\title{
Utilization of a Novel Method of RNA Interference in Caenohabditis elegans to Conduct a Phenotypic Analysis of the daf-2 and daf-16 Longev- ity Genes
}

\author{
Rincon Jagarlamudi \\ Marquette High School, 2351 Clarkson Road, Chesterfield, Missouri, 63005, U.S.A.; rjagarlamudi043@rsdmo.org
}

\begin{abstract}
The daf-2 and daf-16 genes have been highlighted for their reciprocal activity in altering longevity in the model organism Caenorhabditis elegans. Both daf-2 and daf-16 present orthologs in the human genome that maintain similar function. The most straightforward method for analyzing the impacts of these genes on worm phenotype is through RNA interference. This can be accomplished by feeding the worms Escherichia coli containing double stranded RNA intended to make the target gene inoperative in the nematodes. However, as the worms age, consumed E. coli colonizes within the gut, resulting in a pathogenic infection. As part of the following research, a novel procedure for RNA interference with Bacillus subtilis was tested and implemented to analyze the IIS signaling pathway. Using this novel system, the daf-2 and daf-16 genes in the IIS pathway were analyzed and compared with the results yielded through RNA interference with $E$. coli. The results from the study indicate reduced bending and maneuverability among daf-2 mutants suggesting that successful interference was achieved. Furthermore, worms that underwent $\mathrm{B}$. subtilis $\mathrm{RNAi}(\mathrm{M}=.8290, \mathrm{SE}=0.026)$ are more likely to live longer $\left(\mathrm{t}_{(68)}=-4.402, \mathrm{p}=.000\right)$ than mutants undergoing conventional RNAi using $E$. coli $(\mathrm{M}=.6133$, SE 0.04). In addition, the daf- 2 mutants $(\mathrm{M}=0.8783, \mathrm{SE} 0.0186)$ arrested development and entered the Dauer stage, resulting in a longer lifespan $(\mathrm{t}(68)=7.008, \mathrm{p}=.000)$ than daf-16 mutants $(\mathrm{M}=$ $0.5640 \mathrm{SE}$ 0.0407). This study demonstrates that using B. subtilis as a feeding vector is the superior course of action for RNAi in C. elegans because $B$. subtilis RNAi is a better representation of the worm's in situ environment and that daf-2 gene inactivation lengthens C. elegans lifespan on a non-pathogenic bacteria (B. subtilis), suggesting the mutation slows aging.

KEYWORDS: Biotechnology; Genetics; Caenorhabditis elegans; Double Stranded RNA Interference; IIS Signaling Pathway; Bacillus subtilis; Escherichia Coli; daf-2; daf-16.
\end{abstract}

- Introduction

In the field of genetics, the organism Caenorbabditis elegans (C. elegans) presents a powerful model to understand human genetics. This is due to the fact that $83 \%$ of the genes expressed in C. elegans corresponded to human genes. ${ }^{1}$ Therefore, the knowledge about the $C$. elegans' genome will also provide considerable insight about the human genome that can be applied to study various disorders such as Alzheimer's and Parkinson's Diseases that present a genetic predisposition.

To study the C. elegans' genome, RNA interference (RNAi) method is implemented through the usage of double stranded RNA. ${ }^{2}$ The double stranded RNA is readily available to this organism through a diet that is composed primarily of bacterial sources. The most common feeding vector for this procedure is OP50 E. coli due to its ready availability and its limited growth rate. The consumption of such double stranded RNA performs a "knockout" of gene targets through a post-transcriptional mechanism. However, concerns over this practice have arose due to $E$. coli's ability to colonize in the worms' gut. Several reports have found that once the worms reach old age, the colonization of their intestine by the $E$. coli degrades the intestinal villi allowing the $E$. coli to spread throughout the worm. ${ }^{3,5}$ The bacterial pathogenicity found in $E$. coli has led to concerns surrounding gene screenings that utilize RNAi with the bacteria as a feeding vector.
Accordingly, another system for RNAi screening in C. elegans using different bacterium, Bacillus subtilis (B. subtilis), has been identified. One of the bacteria that comprise the microbiota of these regions is B. subtilis. This bacterium is commonly found in the $C$. elegans' environment: areas with decaying plant material. Unlike E. coli, B. subtilis would better represent the worm in situ, or similar to what is experience in their natural environment. Surprisingly, when the lifespans of worms grown on $B$. subtilis were compared to those grown on $E$. coli, the worms growing on $B$. subtilis lived 50\% longer than those living on $E$. coli. This difference in host longevity is likely due to a difference in pathogenicity between the two bacteria. Although B. subtilis colonizes in the nematode's gut, just like $E$. coli, it produces a protective biofilm, which produces two essential products: the quorum-sensing molecule CSF and nitric oxide. These compounds are the primary contributors to the extension of the worm's lifespan. ${ }^{3}$

Wild type (WT) B. subtilis presents a $\mathrm{rncS}$ gene that codes for an endoribonuclease that cleaves dsRNA, which makes the strains containing the gene not practical for gene screening with RNAi because the introduction of dsRNA is necessary for this process. ${ }^{6}$ Another challenge that was presented as a result of using B. subtilis as a feeding vector for RNAi was that natural isolates of $B$. subtilis present low genetic competence, meaning that they had a reduced capacity for taking up 
genetic material. As a result, the conventional method for bacterial transformation could not be applied for this experiment. Without the expression of the dsRNA found in the plasmid, the gene targets cannot be "knocked out" in C. elegans to be studied. Thus, to overcome these limitations, the RNase IIInull BG322 mutant strain and a novel protocol for bacterial transformation that employed the sugar xylose for increasing genetic competence were used for the present study. The BG322 mutant subdues the rncS gene, thereby allowing for standard uptake of the plasmid. ${ }^{7}$

The present study investigates the effects of $d a f-2$ and daf16 gene knockouts on the lifespan and locative effects of $C$. elegans using a novel system for RNAi that utilizes B. subtilis as a feeding vector. Both the daf-2 and daf-16 genes are components of the IIS signaling pathway, which a major contributor to the worm lifespan. The IIS signaling pathway consists of the $D A F-16$ protein, $D A F-2$ protein, and the serine/threonine kinases: PDK-1, AKT-1, and AKT-2. When the $D A F-2$ protein is activated, these three kinases are found upstream of DAF16 , and possess the capability to phosphorylate the DAF-16 protein and prevent its entrance into the nucleus, whereas $D A F-16$ would normally activate genes that promote the longevity of $C$. elegans, thereby allowing the kinases to control lifespan. ${ }^{8}$

Implementing a novel method of RNA interference for the analysis the IIS pathway could lead to new discoveries about the pathway and its role in C. elegans lifespan.

\section{Results and Discussion}

\section{Longevity Assay}

B. subtilis was transformed using plasmid vectors obtained from Addgene, containing different genes of interest targeting the daf-2 and daf-16 genes. The pAD48-daf-2 RNAi plasmid contains the daf-2 gene and the pAD43-daf15 RNAi plasmid introduces the daf-16 gene into the B. subtilis. Once the $C$. elegans consumed the B. subtilis and E. coli containing the daf2 or $d a f-16$ genes, the RNAi process deactivated these genes respectively within their genomes. Following this process, I performed a lifespan analysis of the mutated worms, comparing their lifespans in regard to their food source and their gene mutation. The worms grown on B. subtilis lived longer, on average, than their counterparts grown on $E$. coli, regardless of their genetic mutation. The daf-2 mutants grown on B. subtilis lived, on average, $26 \%$ longer than those grown on E. coli. This difference, however, was more pronounced among the daf-16 mutants because those grown on B. subtilis lived, on average, $59 \%$ longer that those grown on $E$. coli. This indicates that the daf- 2 mutants are either less susceptible to bacterial pathogenesis or that daf-16 mutants are more susceptible to bacterial colonization within the gut. Next, I compared the lifespans of the daf-2 and daf-16 mutants on B. subtilis to the wildtype nematodes grown on the same food source, which acted as a control. Interestingly, the average lifespan of the daf-2 and daf-16 mutants were respectively longer and shorter than the average lifespan on the $\mathrm{N} 2$ worms. This suggests that the daf-2 gene inactivation increases lifespan whereas the daf-16 gene inactivates decreases lifespan.
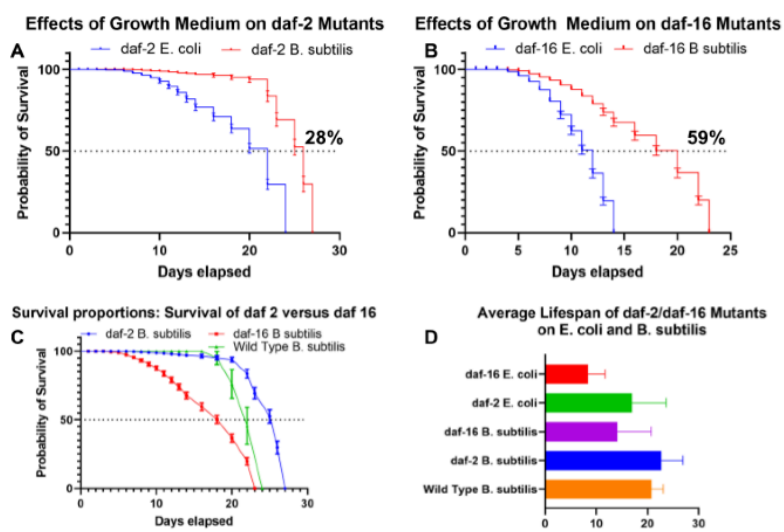

Figure 1. (A) daf 2 mutants fed endoribonuclease-null B. subtilis (BG322) lived 28\% longer compared to those fed the laboratory $C$. elegans food strain (OP50). The worms experiencing daf-2 RNAi on B. subtilis (BG322) did not display a significant difference in lifespan when compared to those fed E. coli (OP50; $\mathrm{p}=0.087>0.05)$. (B) daf-16 $\mathrm{mu}^{-}$ tants fed endoribonuclease-null B. subtilis (BG322) lived 28\% longer compared to those fed the laboratory $C$. elegans food strain (OP50). The worms experiencing daf-16 RNAi on $B \cdot$ subtilis displayed a significant difference in lifespan when compared to those fed $E$ coli ( $\mathrm{p}<0.05)$. (C) daf-2 mutants grown on the BG322 B. subtilis strain lived $41 \%$ longer than daf-16 mutants fed BG322 B. subtilis ( $\mathrm{p}<0.05$ ). (D) Graph displays the average lifespan of the experimental groups. Median lifespan: daf-16 on E. coli $=12$ days, daf -16 on $B \cdot$ subtilis $=20$ days, daf -2 on E. coli $=22$ days, and daf -2 on B. subtilis $=26$ days. Age refers to the number of days after egg hatching. Two biological replicates were observed for all mutant nematodes $(n=59-82$ worms per experiment); errors bars designate standard error.

\section{Locomotion Assay}

In addition to worm lifespan, an important measurement of worm phenotype is worm locomotion. This phenotypic quality is particularly important because it can uncover the mecha ${ }^{-}$ nisms by which gene mutations alter phenotype. To analyze worm locomotion, a software called WormLab was utilized. This software analyzed 30 second videos of C. elegans and $\mathrm{cre}^{-}$ ated a locomotion summary. Worm bending and displacement were determined respectively by the maximum amplitude and peristaltic track length. The results indicate that daf-2 mutants present reduced locomotive displacement and bending.
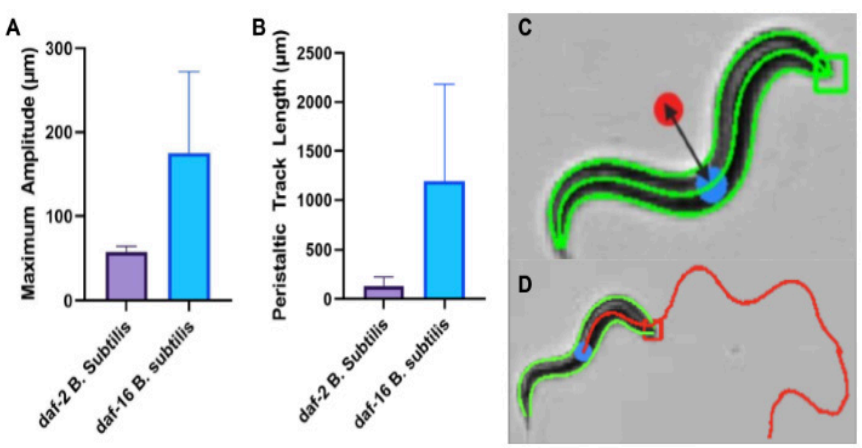

Figure 2. This figure shows the reduced motility and bending of daf-2 RNAi mutants in comparison to the daf-16 RNAi as shown by the (A) maximum amplitude and (B) peristaltic track length. $\mathrm{N}=4$ per experimental group. (C) The figure above illustrates the method for calculating maximum bending amplitude. The maximum amplitude is calculated by determining where the worm's centroid displacement is greatest. Centroid displacement can be calculated by determining the distance between the worm's midpoint and the average location of the central axis. (Blue = Midpoint; Red= Central Axis). (D) The figure above illustrates the method for calculating peristaltic track length, The peristaltic track length is calculated by calculating the length of the red line in the picture.

\section{Longevity Assay Analysis and Discussion}

As emphasized by Uno and Nishida, the daf-2 and daf-16 genes reciprocally affect $C \cdot$ elegans lifespan. Both of these genes 
operate in the IIS pathway, which plays a large role in regulat ${ }^{-}$ ing the lifespan of $C$. elegans. ${ }^{9}$ The decrease in daf-2 activity, which can be accompanied by a two-fold increase in lifespan, is dependent on an increase in daf-16 activity. Subsequent ly, daf-16 maneuvers to the nucleus where it modifies genes that regulate metabolism, the cellular stress response, and $\mathrm{au}^{-}$ tophagy. ${ }^{10}$ As a result, the extension of lifespan induced by the knockout of daf-2 and the shortening of lifespan generated by the knockout of daf-16 coincides with previous research. After nine days since hatching, $28 \%$ more of the $C$. elegans feeding on daf-2 B. subtilis are alive in comparison to those feeding of daf-16 B. subtilis.

When analyzing the impact of the C. elegans' feeding vector on the lifespan of worms undergoing daf-16 RNAi, there was a significant decline in lifespan for worms with an $E$. coli diet in comparison to those with a B. subtilis diet. However, when examining the worms experiencing daf-2 RNAi, it was surprising to find that there was no longer a significant difference in C. elegans longevity. This contrast is likely in part due to the mechanisms by which the daf-2 and daf- 16 genes alter lifespan.

The FOXO transcription factor daf-16 is a key regulator of the C.elegans' innate immune system due to its close relationship with the protein FOXO3a, which controls the inflammation that accompanies a bacterial infection. ${ }^{11}$ This would further exacerbate the colonization of the worm's gut, which is speculated to be a consequence of a weakened immune system as the worm grows older. As a result, the knockout of the daf- 16 gene prevents this regulation of the immune system, leaving the nematode more susceptible to E. coli colonization within the intestinal lumen and the subsequent migration to adjacent tissues, which increases worm mortality.

Even when food is present, daf-2 loss of function mutants frequently enter the Dauer life stage, which is intended for worms facing a harsh environment. ${ }^{12}$ This behavior is likely due to the elevated sensory perception in the worms, which is provoked by increased expression of the odr-10 receptor gene. As highlighted by Podshivalova et. al, by examining C. elegans consuming GFP expressing OP50 E. coli, daf-2 mutants are more resistant to bacterial pathogenicity, which would account for the lack of difference in longevity for daf-2 mutants feeding on E. coli and B. subtilis. ${ }^{14}$ Although the exact mechanism by which daf-2 mutants are resistant to bacterial colonization is not fully understood, this behavior can possibly be explained by higher expression of antimicrobial genes. ${ }^{15}$ Pronounced resistance among daf-2 mutants would mitigate the lifespan decline commonly induced by feeding on $E$. coli, considering the primary factor that is contrasting between $B$. subtilis and $E$. coli is pathogenicity.

\section{Locomotion Assay Analysis and Discussion}

As demonstrated by the elevated bending amplitude (Figure $2 \mathrm{~A}$ ) and peristaltic track length (Figure $2 \mathrm{~B}$ ), the daf-16 mutants exhibit greater motility in comparison to their daf-2 mutants. This differentiation between the two mutants is likely a conse ${ }^{-}$ quence of the enhanced ability for daf-2 mutants to enter the Dauer stage. ${ }^{16,17}$ While in the Dauer phase, worm locomotion is remarkably reduced due to a decline in the pumping of the worm's pharynx. This is likely because the mutant's resources are greatly devoted to developing the worm's immune system.

\section{Conclusion}

As shown in this study, the decline in lifespan caused by daf16 mutation is worsened in E. coli in comparison to B. subtilis. In order to identify if this decline in lifespan for daf-16 mutants on OP50 E. coli is due to a weakened immune system, the nematodes can be fed GFP expressing OP50 E. coli. The rate of fluorescence in adjacent tissues could then be recorded using fluorescence microscopy and subsequent analysis of ROIs. In addition, to analyze the effects of daf-16/daf-2 RNAi with $B$. subtilis on worm offspring, the remaining worms can be bleached to leave the eggs on the test plates. Then, the number of eggs and genders of the offspring can be analyzed.

Although the IIS signaling pathway plays a large role in regulating lifespan, the TOR pathway is also a significant regulator as well. This pathway is primarily regulated by the PHA-4/ FoxA transcription factor instead of the $D A F-16 / \mathrm{FOXO}$ factor in IIS signaling. A gene that regulates this pathway is the rsks- ${ }^{-}$ gene, which could also be knocked out using B. subtilis RNAi.

Although the results from this research presents a means for reducing lifespan with a daf-16 mutation, it is more difficult to increase lifespan in daf-2 mutants due to the limiting effects of the gene on bacterial colonization. Furthermore, this leaves the nematodes in a prolonged state of nutrient restriction and bacterial colonization in the intestine regardless of the bacteria because the daf-2 gene conducts initiate the entry of the worms into Dauer stage. Nevertheless, this study has shown that $B$. subtilis RNAi is likely superior over conventional RNAi because its colonization in the worms is less pathogenic in comparison to E. coli colonization.

By utilizing daf-2 RNA interference, researchers can increase the lifespan of $\mathrm{C}$. elegans. This capability to increase the nematodes lifespan will allow researchers to study diseases that occur later in the organism's lifespan, providing information beneficial in studying conditions in the C. elegans similar to Alzheimer's and Parkinson's Disease in humans. However, this technique cannot be utilized to study locomotive disorders because the locomotive assay from this project found that daf-2 mutants presented limited motility, which prevents an accurate representation of the deactivated gene's impact on locomotion.

\section{Methods}

\section{Analysis}

For this research, the longevity of the C. elegans was measured for the nematodes that undergo the RNA interference with $B$. subtilis and those that undergo RNA interference using $E$. coli. An ANOVA statistical test was run to determine the difference of longevity between the two sets of nematodes. The data was also analyzed using a Kaplan Meier statistical analysis and a Tukey post-hoc test.

\section{Materials}

The 10-microliter sample of BG322 Bacillus subtilis bacteria necessary for this experiment was provided by Dr. David Be- 
chhofer at the Mount Sinai Hospital in New York, New York. The wild type C. elegans were provided by BRDG Park Facility at the Danforth Center and Dr. Zachary Pincus's lab at Washington University in St. Louis. The plasmids necessary to knockout the $D A F-16$ and $D A F-2$ genes (pAD48-daf-2 and pAD43-daf-16) came from the nonprofit plasmid repository Addgene. Other general lab materials were found either at Marquette High School or the Pincus Lab facility.

\section{Plasmid Preparation}

The plasmids were shipped by the Addgene repository in the form of an agar stab with transformed $E$. coli containing the plasmid. In order to prepare transformed B. subtilis, the plasmid was extracted prior to the transformation of the B. subtilis. In order to isolate the dsRNA from the $E$. coli, the cells were lysed in order to expose the nuclear contents for extraction. Then, the solution was rapidly acidified with the goal of separating proteins and chromosomal DNA from plasmid DNA. A potassium acetate solution was used for this purpose because it precipitates proteins and chromosomal DNA, but the plasmid DNA remains in solution. The plasmid DNA, which remains in the supernatant, can then be captured by a silica spin column and then eluted using water or TE buffer. ${ }^{18}$

\section{Transformation}

Naturally isolated B. subtilis presents low genetic competence, which is defined as an organism's ability to take up exogenous DNA. As a result, it is necessary to use alternative methods when transforming $B$. subtilis. The cells were first centrifuged at $8,000 \mathrm{rpm}$ for 1 minute and the supernatant was discarded. Then 200 microliters of an LB broth medium and $2 \%$ xylose solution was added. The appropriate plasmid DNA was introduced to the cell suspension and then nutated in an incubator set at 37 degrees Celsius overnight. ${ }^{24}$

\section{Worm Synchronization}

When conducting longevity assays, it is crucial to synchronize all the worms being studied at the larvae stage. This ensures there is accurate calculation of surviving worms on each plate. Washing C. elegans with a sodium hydroxide and hypochlorite solution degrades gravid worms and leaves the eggs intact. The yield of eggs that remain can be suspended in a synchronization medium (M9 buffer) and then pipetted onto plates containing the transformed bacteria. Because all the C. elegans were eggs at the initial phase of data collection, it was ensured that all the C. elegans were at the same life stage at a specified time.

\section{Analysis of B. subtilis RNAi Viability:}

During the first year of this project, bacterial transformation of the plasmid was monitored in B. subtilis, by using a novel system, which relies on $\beta$-galactosidase $\alpha$-complementation. Following the transformation of the B. subtilis, the bacterial culture was added to IPTG (Isopropyl $\beta$ - d-1-thiogalactopyranoside) agar plates. This compound mimics allolactose, which can initiate lac operon transcription by binding to and altering the shape of the lac repressor. IPTG can be used in conjunction with the $\mathrm{X}$ - gal substrate (5-bromo-4-chloro-3-indolyl- $\beta$-D-galactopyranoside) as part of the blue-white screening method in order to detect recombinant bacteria. C. elegans were chunked into small sections and placed onto the IPTG plates with the Bacillus subtilis. The worms were then periodically analyzed to look for any signs of a die off that may have been caused by the new diet of Bacillus subtilis. The presence of blue fluorescing colonies on the plates would indicate the presence of functional $\beta$-galactosidase, which indicates those bacterial colonies took up the plasmid and contains recombinant DNA. ${ }^{19}$

\section{Phenotypic Analysis of Transformed Nematodes}

The software WormLab was used to conduct a locomotion analysis of the nematodes that are being studied. In addition to analyzing locomotion, this software can accurately track complex movements such as entanglements, coiling, and omega bends. ${ }^{26,27}$ Prior to analyzing the video file of the C. elegans, all frames underwent background smoothing and the threshold level was adjusted, so the worms were detectable by the software. Gaussian smoothing was avoided in order to maintain resolution.

\section{Acknowledgements}

This study's success is due, in part to the work of the author's Authentic Science Research teacher Dr. Cathy Farrar. She has been of great help in the past year in terms of ordering necessary materials and providing lab space for the project. Also, the author like to thank Mrs. Boedeker at the STLCC BRDG Park facility for providing the C. elegans during his first year of research. The Pincus Lab at Washington University in St. Louis has been supportive in allowing us to use their digital microscopes for the locomotion assay. Dr. Zach Pincus's help has been greatly appreciated. Dr. David Bechhofer was instrumental in this project's success by providing the BG322 strain of $B$. subtilis, without which this project could not have been possible.

\section{References}

1. Lai, C., 2000. Identification of Novel Human Genes Evolutionarily Conserved in Caenorhabditis elegans by Comparative Proteomics. Genome Research, 10(5), pp.703-713.

2. Fire, A., Xu, S., Montgomery, M., Kostas, S., Driver, S. and Mello, C., 1998. Potent and specific genetic interference by double-stranded RNA in Caenorhabditis elegans. Nature, 391(6669), pp.806-811.

3. Donato, V., Ayala, F., Cogliati, S., Bauman, C., Costa, J., Leñini, C. and Grau, R., 2017. Bacillus subtilis biofilm extends Caenorhabditis elegans longevity through downregulation of the insulin-like signalling pathway. Nature Communications, 8(1).

4. Félix, M. and Duveau, F., 2012. Population dynamics and habitat sharing of natural populations of Caenorhabditis elegans and C. briggsae. BMC Biology, 10(1).

5. Garsin, D., 2003. Long-Lived C. elegans daf-2 Mutants Are Resistant to Bacterial Pathogens. Science, 300(5627), pp.1921-1921.

6. Herskovitz, M. and Bechhofer, D., 2002. Endoribonuclease RNase III is essential in Bacillus subtilis. Molecular Microbiology, 38(5), pp.1027-1033.

7. Durand, S., Gilet, L., Bessières, P., Nicolas, P. and Condon, C., 2012. Three Essential Ribonucleases-RNase Y,J1, and III-Control the Abundance of a Majority of Bacillus subtilis mRNAs. PLoS Genetics, 8(3), p.e1002520.

8. Dillin, A., 2002. Timing Requirements for Insulin/IGF-1 Signaling in 
C. elegans. Science, 298(5594), pp.830-834.

9. Uno, M. and Nishida, E., 2016. Lifespan-regulating genes in C. elegans. npj Aging and Mechanisms of Disease, 2(1).

10.Podshivalova, K., Kerr, R. and Kenyon, C., 2017. How a Mutation that Slows Aging Can Also Disproportionately Extend End-of-Life Decrepitude. Cell Reports, 19(3), pp.441-450.

11. Tao, L., Xie, Q., Ding, Y., Li, S., Peng, S., Zhang, Y., Tan, D., Yuan, Z. and Dong, M., 2013. CAMKII and Calcineurin regulate the lifespan of Caenorhabditis elegans through the FOXO transcription factor DAF-16. eLife, 2.

12. Fielenbach, N. and Antebi, A., 2008. C. elegans dauer formation and the molecular basis of plasticity. Genes \& Development, 22(16), pp.2149-2165.

13.Gems, D., Sutton, A., Sundermeyer, M., Albert, P., King, K., Edgley, M., Larsen, P. and Riddle, D., 1998. Two pleiotropic classes of daf- 2 mutation affect larval arrest, adult behavior, reproduction and 1 ongevity in Caenorhabditis elegans. Genetics, 150(1), pp.129-155.

14.Singh, V. and Aballay, A., 2009. Regulation of DAF-16-mediated Innate Immunity in Caenorhabditis elegans. Journal of Biological Chemistry, 284(51), pp.35580-35587.

15.Murphy, C., Mccaroll, S., Bargmann, C., Fraser, A., Kamath, R., Ahringer, J., Li, H., Kenyon, C., 2003. Genes That Act Downstream of DAF-16 to Influence the Lifespan of Caenorhabditis Elegans. Nature, 424 (6946), pp. $277-283$.

16.Angstman, N. B., Kiessling, M. C., Frank, H.-G., Schmitz, C., 2015, High Interindividual Variability in Dose-Dependent Reduction in Speed of Movement after Exposing C. Elegans to Shock Waves. Frontiers in Behavioral Neuroscience, 9.

17.Ehrt, S., Schnappinger, D., 2003. Isolation of Plasmids from E. Coli by Alkaline Lysis. E. coli Plasmid Vectors, pp.75-78.

18.Zhang, X. and Zhang, Y., 2010. Simple, fast and high-efficiency transformation system for directed evolution of cellulase in Bacillus subtilis. Microbial Biotechnology, 4(1), pp.98-105.

19.Sambrook, J., Russell, D. W., 2006. Screening Bacterial Colonies Using X-Gal and IPTG: a-Complementation. Cold Spring Harbor Protocols, 2006 (1).

\section{Author}

My name is Rincon Jagarlamudi and I am currently a junior attending Marquette High School in St Louis, Missouri. I enjoy learning from all fields of science but particularly enjoy the fields of neuroscience, genetic engineering, and chemistry. Currently, my career path is undecided; however, scientific research is a potential field of interest. 